\title{
Solubility Prediction of Some Disperse Azo Dyes in Supercritical Carbon Dioxide using Equation of States (EOSs)
}

\author{
Shahryar Jafari Nejad*, Milad Asgarpour Khansary, Farshad Amiri \\ School of Chemical Engineering, College of Engineering, University of Tehran, Tehran, Iran \\ Caspian Faculty of engineering, College of Engineering, University of Tehran, Iran \\ "E-mail: shjafarinejad@ut.ac.ir
}

\begin{abstract}
The solubility of three disperse azo dyes, 4-(N,N-dimethylamino)-4'-itroazobenzene ( $\left.\mathrm{D}_{1}\right), 4$-(N,N-diethylamino)-4'nitroazobenzene $\left(\mathrm{D}_{2}\right)$ and Parared $\left(\mathrm{D}_{3}\right)$ in supercritical carbon dioxide have been correlated with two equations of state. All critical properties have been estimated with a group contribution method (GCM). As far we know, solubility data for these dyes have never been correlated using an equation of state (EOS). Therefore, it is worthwhile to model the solubility of these disperse Azo dyes. In this work, the aim is correlating reported data with a new (M-factor) EOS and comparing obtained results with the results of Peng-Robinson EOS (PR-EOS) together with two adjustable parameter van der Waals mixing and combining rules. The calculated results showed that Mfactor EOS is more accurate than PR-EOS. It can be employed to speed up the process of supercritical fluid (SCF) applications in industry.
\end{abstract}

Keywords: Solubility; disperse azo dyes; supercritical carbon dioxide; correlation; equation of state (EOS).

\section{Introduction}

In the past decades, there has been an increasing interest in the use of supercritical fluids as an alternative to the use of organic solvents in many industrial applications, such as in chemical and biochemical reactions, extraction and purification processes, particle production, textile industry, etc. (Teja et al, 2000, Jafari Nejad et al, 2010a, 2010b, 2010c, Jung et al, 2001, Cooper et al, 2003, Kazarian et al, 2000, Kikic et al, 2003).

Supercritical carbon dioxide is the most commonly used supercritical fluid. The critical temperature and pressure of carbon dioxide is relatively low $(304 \mathrm{~K}$ and $73.7 \mathrm{bar}$, respectively) (Jafari Nejad et al, 2009) and one of the most environmentally acceptable solvents in use today, and textile processes using this solvent have many advantages when compared to conventional aqueous processes (Fasihi et al, 2004). Supercritical carbon dioxide gives an option avoiding water discharge, it is low in cost, nontoxic, and nonflammable, and the carbon dioxide can be recycled. Also, when dying from an aqueous medium, reduction clearing is carried out to stabilize the color intensity, producing further water waste. Reduction clearing is not carried out following supercritical dyeing. Supercritical carbon dioxide also has other advantages. The application of the dye to the fabric can be controlled and a better quality of application achieved (Saus et al, 1995, Clifford et al, 1996). The dyes used in supercritical dyeing are the nonionic, so-called disperse dyes.

To develop and design the supercritical fluid dyeing (SFD) process a lot of basic dye solubility data and modeling of these solubility data are necessary. Disperse Dyes for dyeing polyester textile is divided into two groups: Azo and anthraquinone derivatives (Bae et al, 1996, Haarhaus et al, 1995, Joung et al, 1998).

In the mathematical modeling of solubility data in supercritical fluids, one should keep in mind that the solubility systems can be categorized in three groups, a single solute in a supercritical fluid, mixed solutes in a supercritical fluid and a single solute in mixed supercritical fluids or supercritical fluid plus an organic solvent. Different equations have been presented for mathematical modeling of solubility data in $\mathrm{SC} \mathrm{CO}_{2}$. One can categorize these models into two groups, theoretical or semi-empirical equations (similar to models based on equations of state) and empirical equations (such as density based equations). Models derived from equations of state need complicated computational procedures that are not provided in commonly used commercial software. Also, these models employ the solute properties, such as critical properties, acentric factor, molar volumes and vapor pressure, which often cannot be easily determined experimentally. The numerical values of the solute properties can affect solubility predictions using models derived from equations of state [2]. To avoid some of these difficulties as well as more complicated computational routines, most authors opt to use empirical correlations such as density-based correlations (Chrastil, Bartle, M'endez-Santiago-Teja, Jafari Nejad et al. and etc. models), or the Ziger-Eckert semi-empirical correlation. These models are based on simple error minimization using least-squares methods, and for the majority of them, there is no need to estimate and use critical and thermophysical properties of the involved solutes (Jafari Nejad et al, 2009, 2010).

In this study, solubilites of three disperse Azo dyes, 4(N,N-dimethylamino)-4'-nitroazobenzene $\quad\left(\mathrm{D}_{1}\right), \quad 4-(\mathrm{N}, \mathrm{N}-$ diethylamino)-4'-nitroazobenzene $\left(\mathrm{D}_{2}\right)$ and Parared $\left(\mathrm{D}_{3}\right)$ (Fasihi et al, 2004) in supercritical carbon dioxide have been correlated with two equation of state; Peng-Robinson EOS (PR-EOS) together with two adjustable parameter van der Waals mixing and combining rules and new (M-factor) EOS (Jafari Nejad et al, 2009). As far we know, solubility data for these dyes never has been correlated using an 
equation of state (EOS). Therefore, it is worthwhile to model the solubility of these disperse Azo dyes.

\section{Theory}

The use of different models to correlate the solubility data was investigated using models based on a cubic EOS (Peng-Robinson (PR-EOS); together with two adjustable parameter van der Waals ( $\mathrm{vdW}$ ) mixing and combining rules and a new equation of state (EOS).

\subsection{Peng-Robinson equation of state (PR-EOS)}

The solubility of a solute $\left(\mathrm{y}_{2}\right)$ at equilibrium with a fluid, at high pressures, can be calculated using the following expression:

$$
y_{2}=\frac{P_{2}^{\text {sat }}}{P} \frac{1}{\phi_{2}^{\text {sCF }}} \exp \left[\frac{v_{2}\left(P-P_{2}^{\text {sat }}\right)}{R T}\right]
$$

Here, $P_{2}{ }^{\text {sat }}$ is the saturation pressure of the solute, $v_{2}$ the molar volume of the solute and $\varphi_{2}{ }^{S C F}$ is the fugacity coefficient of the solute in the fluid phase, which expresses the non-ideality of the fluid phase. The fugacity coefficient can be calculated with an equation of state. In this work, the Peng-Robinson EOS (Eqs. (2) - (5)), here defined for pure substances, were used:

$$
\begin{aligned}
& P=\frac{R T}{v-b}-\frac{a}{v(v+b)+b(v-b)} \\
& a=0.45724\left(\frac{R^{2} T_{c}^{2}}{P_{c}}\right)\left\{1+n\left[1-\left(\frac{T}{T_{c}}\right)^{0.5}\right]\right\}^{2}
\end{aligned}
$$

$n=0.37464+1.54226 \omega-0.26992 \omega^{2}$

$$
b=0.07780 \frac{R T_{c}}{P_{c}}
$$

To use the above EOS for a binary mixture, we employed the classical van der Waals (vdW) mixing and combining rules, with two adjustable parameters, $\mathrm{k}_{\mathrm{ij}}$ and $\mathrm{l}_{\mathrm{ij}}$ (vdW2):

$$
\begin{aligned}
& a=\sum_{i} \sum_{j} y_{i} y_{j}\left(a_{i} a_{j}\right)^{0.5}\left(1-k_{i j}\right) \\
& b=\sum_{i} \sum_{j} y_{i} y_{j}\left(\frac{b_{i}+b_{j}}{2}\right)\left(1-l_{i j}\right)
\end{aligned}
$$

The binary interaction parameters, $\mathrm{k}_{\mathrm{ij}}$ and $\mathrm{l}_{\mathrm{ij}}$, are obtained by fitting experimental data, through the minimization of an objective function (Robinson et al, 1976, Coimbra et al, 2005).

Using the conventional mixing rules for a mixture, the fugacity coefficient for component $\mathrm{h}$ in a mixture is given by (Prausnitz et al, 1999):

$$
\begin{aligned}
\operatorname{Ln} \phi_{h} & =\frac{b_{k}}{b}\left(\frac{P v}{R T}-1\right)-\operatorname{Ln} \frac{P(v-b)}{R T} \\
& -\frac{a}{2 \sqrt{2} b R T}\left[\frac{2 \sum_{i} y_{i} a_{i h}}{a}-\frac{b_{h}}{b}\right] \operatorname{Ln} \frac{v+(1+\sqrt{2}) b}{v+(1-\sqrt{2}) b}
\end{aligned}
$$

Where, $y_{i}$ is the mole fraction of component $i$.

The individual absolute relative deviations (IARD) of calculated solubilities from observed values are used as an accuracy criterion to compare the calculated solubilities with experimental values. IARD was calculated by:

$\operatorname{IARD}(\%)=100\left(\frac{\left|y_{2}^{\text {cal }}-y_{2}^{\exp }\right|}{y_{2}^{\exp }}\right)$

The average-absolute-relative-deviation

(AARD) defines as:

$$
\operatorname{AARD}(\%)=\frac{100}{N} \sum \frac{\left|y_{2}^{c a l}-y_{2}^{\text {exp }}\right|}{y_{2}^{\exp }}
$$

In this equation, $N$ is the number of experimental data points, $y_{2}{ }^{\text {cal }}$ and $y_{2}{ }^{\text {exp }}$ are calculated solubilities and experimental solubility, respectively.

\subsection{Theory of new (M-factor) equation of state}

The virial EOS was originally introduced by Kammerligh-Onnes as of ascending power of density to represent the compressibility factor. Later on, Ursell and Mayer (1957) developed the statistical mechanic for virial equation, which is formally presented as a series expansion of either the radial distribution function or the grand canonical partition function for low-density gases. The virial coefficients are related to the intermolecular potential energy so that B is linked through rigorous relations to the so-called pair potential energy function, which is responsible for many thermodynamic and transport properties of fluid (Vetere, 1999), C is related to the energy of interaction between triples of molecules, and so forth. The Leiden virial equation of state gives the compressibility factor as a power series in the reciprocal molar volume:

$Z=\frac{P v}{R T}=1+\frac{B}{v}+\frac{C}{v^{2}}+\frac{D}{v^{3}}+\ldots$

The mathematically analogous power series in the pressure can be derived from Eq.11 and is known as the Berlin virial EOS:

$Z=\frac{P v}{R T}=1+B^{\prime} P+C^{\prime} P^{2}+D^{\prime} P^{3}+\ldots$

The molar volume in Eq.12 was explicitly obtained and then substituted into Eq.11. Hence two set of coefficients in Eq. 11 and Eq.12 are related as below:

$$
\begin{aligned}
& B^{\prime} \approx \frac{B}{R T} \quad C^{\prime} \approx \frac{C-B^{2}}{(R T)^{2}} \quad D^{\prime} \approx \frac{D-3 B C+2 B^{3}}{(R T)^{3}} \\
& E^{\prime} \approx(R T)^{4}\left(\frac{E-5 B^{4}-2 C^{2}-4 B D}{(R T)^{4}}+10 B^{2} C\right)
\end{aligned}
$$

Because the C, D, E and higher virial coefficients are responsible for molecular interactions, thus these are generically dependent on binary interactions. From Eq.13, 
it is intelligible that whatever the molecular interaction effects become more intense, the second virial coefficient takes higher order of magnitude. For example, $C^{\prime}$ and $D^{\prime}$ are proportional to second and third power of $\mathrm{B}$. Mathematically, there are the same terms of B/RT in all of the above relations. Also, much less have been known the third and fourth virial coefficients than the second virial coefficient though data of third coefficient for certain gases can be found in literature (Johnston et al, 1934, Nicola et al, 2005). Besides, it is feasible that effects of all terms in Eq.13, except B-included, be reconsidered by means of several temperature dependent coefficients. For these reasons, in Eq.13, when the third, fourth and higher order virial coefficients be nonce ignored, Eq.13 diminishes to

$$
\begin{aligned}
& B^{\prime}=\frac{B}{R T}, \quad C^{\prime}=\alpha(T)\left(\frac{B}{R T}\right)^{2}, \\
& D^{\prime}=\beta(T)\left(\frac{B}{R T}\right)^{3}, \quad E^{\prime}=\gamma(T)\left(\frac{B}{R T}\right)^{4}
\end{aligned}
$$

Since the third, fourth and higher order virial coefficients depend only on temperature; several coefficients were inserted behind the relations of Eq.14 to estimate considerable effects of eliminated virial coefficients. These coefficients only have temperature dependency and would make up effects of C, D... which had been removed at previous step. Substituting $B^{\prime}, C^{\prime}, D^{\prime}, E^{\prime}, \ldots$ from Eq.14 into Eq.12 gives

$$
Z=1+\frac{B P}{R T}+\alpha(T)\left(\frac{B P}{R T}\right)^{2}+\beta(T)\left(\frac{B P}{R T}\right)^{3}+\gamma(T)\left(\frac{B P}{R T}\right)^{4}+. .
$$

Eq.15 may be written as

$$
\begin{aligned}
& Z=1+\left[\frac{B P_{c}}{R T_{c}}\right] \frac{P_{r}}{T_{r}}+\alpha(T)\left[\frac{B P_{c}}{R T_{c}}\right]^{2}\left(\frac{P_{r}}{T_{r}}\right)^{2}+\beta(T)\left[\frac{B P_{c}}{R T_{c}}\right]^{3}\left(\frac{P_{r}}{T_{r}}\right)^{3} \\
& +\gamma(T)\left[\frac{B P_{c}}{R T_{c}}\right]^{4}\left(\frac{P_{r}}{T_{r}}\right)^{4}+\ldots
\end{aligned}
$$

Pitzer and Curl (1957) proposed a correlation, which expresses the quantity $\frac{B P_{c}}{R T_{c}}$ as

$$
\frac{B P_{c}}{R T_{c}}=f^{(0)}\left[T_{r}\right]+\omega f^{(1)}\left[T_{r}\right]
$$

The function $f^{(0)}$ gives the reduced second virial coefficients for simple fluids $(\omega=0)$ while $f^{(1)}$ is a correction function which, when multiplied by $\omega$ gives the effect of eccentricity on the second virail coefficient. The two functions $f^{(0)}$ and $f^{(1)}$ were determined from experimental data and modified by Tsonapaulos (1975). Meng et al. (2004) presented a modified corresponding correlation that compares well with experimental data for the second virial coefficient for most non-polar pure compounds, since the predictions have been corrected for most physics effects such as adsorption. Detailed comparisons with the well-known Tsonopoulos correlation showed that the model is somewhat better than Tsonopoulos correlation for non-polar substances. The correlation for non-polar fluids is:

$$
\begin{aligned}
f^{(0)}\left[T_{r}\right]= & 0.13356-\frac{0.30252}{T_{r}}-\frac{0.15668}{T_{r}^{2}}-\frac{0.00724}{T_{r}^{3}} \\
& -\frac{0.00022}{T_{r}^{8}} \\
f^{(1)}\left[T_{r}\right]= & 0.17404-\frac{0.15581}{T_{r}}+\frac{0.38183}{T_{r}^{2}}-\frac{0.44044}{T_{r}^{3}} \\
& -\frac{0.00541}{T_{r}^{8}}
\end{aligned}
$$

For slightly polar substances it is better to utilize Tsonopoulos correlation. Also for polar substances, the second virial coefficient may be calculated from Janecek et al. (2003) correlation or Pires et al. (2001) correlation. For mixtures, the mixing second virial coefficient can be usually predicted with the help of mixing rules. The binary second virial coefficient, for example, is given by:

$$
B_{m}=\sum_{i} \sum_{j} y_{i} y_{j}\left(1-S_{i j}\right) B_{i j}
$$

Where $S_{i j}$ is interaction parameter, is obtained by fitting experimental data, through the minimization of an objective function. For the first time Mohebbi and Mohammadikhah (2007) have represented a new EOS based on the virial equation including $\mathrm{M}$-factor, reduced temperature and reduced pressure. The dimensionless form of M-factor is:

$M=\left[\frac{B P_{c}}{R T_{c}}\right]\left(\frac{P_{r}}{T_{r}}\right)=\frac{B P}{R T}$

With substituting Eq.21 into Eq.15 we get

$Z=1+M+\alpha(T) M^{2}+\beta(T) M^{3}+\gamma(T) M^{4}+\ldots$

As a consequence, this equation explains that the compressibility factor of each substance just depends on Mfactor and temperature. Though $\mathrm{M}$-factor is a compound parameter but can be assumed as a novel parameter with different properties than its composer parameters such as $T_{r}$ or $\mathrm{P}_{\mathrm{r}}$. Thus the compressibility factor can be written as:

$Z=Z\left(M, T_{r}\right)$

From Eq. 22, the modified form can be rewritten as:

$$
Z=f^{1}\left(T_{r}\right)+f^{2}\left(T_{r}\right) M+f^{3}\left(T_{r}\right) M^{2}+f^{4}\left(T_{r}\right) M^{3}
$$

The reason of election third order polynomial versus $M$ is the fitting of this equation all the experimental data with $\mathrm{R}^{2}>0.99$ (Mohebbi et al, 2007, Jafari Nejad et al 2009). Mohammadikhah, Abolghasemi and Jafari Nejad (2009) have developed this EOS for prediction of solute solubility in supercritical carbon dioxide. The coefficients of Eq. 24 are (for $\mathrm{T}_{\mathrm{r}}<1.1$, in this work):

$$
f^{1}\left(T_{r}\right)=1
$$


$f^{2}\left(T_{r}\right)=1$

$f^{3}\left(T_{r}\right)=75.36 T_{r}^{2}-157.7 T_{r}+82.86$

$f^{4}\left(T_{r}\right)=\left|\frac{0.05038 T_{r}+0.0001896}{T_{r}^{3}-2.508 T_{r}^{2}+2.098 T_{r}-0.5845}\right|$

Where these coefficients are for $M \geq-0.39312 T_{r}{ }^{3}+0.0252 T_{r}{ }^{2}-0.001235$, and for $M<-0.39312 T_{r}{ }^{3}+0.0232 T_{r}{ }^{2}-0.001235, \quad$ these coefficients become:

$f^{1}\left(T_{r}\right)=\frac{0.6592 T_{r}^{2}-0.7257 T_{r}+0.208}{T_{r}^{2}-2.4 T_{r}+2.607} \times 0.95$

$f^{2}\left(T_{r}\right)=\frac{729.5 T_{r}^{2}-2499 T_{r}+821.8}{T_{r}^{2}-1836 T_{r}+4545} \times 0.95$

$f^{3}\left(T_{r}\right)=0$

$f^{4}\left(T_{r}\right)=0$

The fugacity coefficient of component $i$ in the fluid mixture is:

$\operatorname{Ln} \hat{\phi}_{i}=\int_{0}^{P} \frac{\left(\bar{Z}_{i}-1\right)}{P} d P$

$P=\frac{M R T}{B} \Rightarrow d P=\frac{R T}{B} d M$

$\operatorname{Ln} \hat{\phi}_{i}=\int_{0}^{M} \frac{\left(\overline{Z_{i}}-1\right)}{M} d M$

The Eq.25 is a multi-domain equation, thus:

$$
\begin{aligned}
\operatorname{Ln} \hat{\phi}_{i} & =\int_{0}^{M_{1}} \frac{\left(\bar{Z}_{i}-1\right)}{M} d M+\int_{M_{1}}^{M} \frac{\left(\overline{Z_{i}}-1\right)}{M} d M \\
& =\left(\operatorname{Ln} \hat{\phi}_{i}\right)_{1}+\left(\operatorname{Ln} \hat{\phi}_{i}\right)_{2}
\end{aligned}
$$

$M_{1}=-0.39312 T_{r}^{\prime 3}+0.0252 T_{r}^{\prime 2}-0.001235$

$T_{r}^{\prime}=T_{r}\left(1-S_{i j}\right)$

The expression for $\varphi_{2}$ is (Jafari Nejad et al, 2009):

$$
\begin{aligned}
\operatorname{Ln} \hat{\phi}_{2} & =\left(\operatorname{Ln} \hat{\phi}_{2}\right)_{1}+\left(\operatorname{Ln} \hat{\phi}_{2}\right)_{2}=\left(f^{1}-1\right) \operatorname{Ln}\left(\frac{M}{M_{1}}\right) \\
& +\frac{f^{2}}{B}\left(M-M_{1}\right) \frac{\partial n B}{\partial n_{2}}+\frac{1}{B} \frac{\partial n B}{\partial n_{2}} M_{1}+\frac{f^{3}}{2 B^{2}} \frac{\partial n B}{\partial n_{2}} M_{1}^{2} \\
& +\frac{f^{4}}{3 B^{3}} \frac{\partial n B^{3}}{\partial n_{2}} M_{1}^{3}
\end{aligned}
$$

Thus the solubility of a liquid solute at equilibrium with a fluid, at high pressures, can be calculated using the Eq.1.

\section{Material and Methods}

The structures of the selected disperse azo dyes and their physicochemical properties are given in Table 1. Fasihi et al. (2004) carry out the solubility measurements of these disperse azo dyes at temperatures 308, 318, 328, 338, 348 and $358 \mathrm{~K}$ over a pressure range from 122 to 355 bar. The experimental results are listed in Table 2 in terms of equilibrium mole fraction, $y$. In order to compare the accuracy of the new correlation with the PR-EOS, it is assumed that all the experimental data are correct. The solubility of $\mathrm{D}_{2}$ is greater than that of $\mathrm{D}_{1}$ and $\mathrm{D}_{3}$ by a factor of about 7 and 20 , respectively. The addition of one $\mathrm{CH}_{2}$ group to $\mathrm{D}_{2}$ molecule, results in a significant solubility enhancement of $\mathrm{D}_{2}$ in comparison with $\mathrm{D}_{1}$ (Fasihi et al, 2004). Similar results have been reported in the literature (Draper et al, 2000).

\begin{tabular}{|c|c|c|c|}
\hline Dyes & Structure & $\begin{array}{c}\text { Melting } \\
\text { point }(K)\end{array}$ & $\begin{array}{c}M_{w} \\
(\mathrm{~g} / \mathrm{mole})\end{array}$ \\
\hline$D_{1}$ & & $488-489$ & 270.12 \\
\hline$D_{2}$ & & $418-419$ & 298.14 \\
\hline $\mathbf{D}_{3}$ & & $529-530$ & 293.13 \\
\hline
\end{tabular}

It should be noted that in this study the program of calculations were written in MATLAB Software by the authors.

Table 1. Structure of dyes and their physicochemical properties (Fasihi et al, 2004) 
Table 2. Correlation and experimental (Fasihi et al, 2004) results of solubilities of three disperse azo dyes, $D_{1}, D_{2}$ and $\mathrm{D}_{3}$ in $\mathrm{SC}-\mathrm{CO}_{2}$ at various temperatures and pressures.

\begin{tabular}{|c|c|c|c|c|c|c|c|c|c|}
\hline \multicolumn{2}{|c|}{ Conditions } & \multicolumn{2}{|l|}{$D_{1}$} & \multicolumn{2}{|l|}{$\mathrm{D}_{2}$} & \multicolumn{2}{|c|}{ Conditions } & \multicolumn{2}{|l|}{$\mathrm{D}_{3}$} \\
\hline $\mathrm{T}(\mathrm{K})$ & $\begin{array}{l}\mathrm{P} \\
\text { (bar) }\end{array}$ & $10^{6} \mathrm{y}$ & $10^{6} \mathrm{y}^{\mathrm{Cal}}$ & $10^{5} \mathrm{y}$ & $10^{5} \mathrm{y}^{\mathrm{Cal}}$ & $\mathrm{T}(\mathrm{K})$ & $\mathrm{P}$ (bar) & $10^{6} \mathrm{y}$ & $10^{6} \mathrm{y}^{\mathrm{Cal}}$ \\
\hline \multirow[t]{9}{*}{308} & 122 & 2.93 & 2.9307 & 0.98 & 0.97830 & \multirow[t]{9}{*}{328} & 122 & 0.32 & 0.3197 \\
\hline & 152 & 2.74 & 2.7407 & 1.12 & 1.1178 & & 152 & 0.48 & 0.4802 \\
\hline & 182 & 2.61 & 2.6070 & 1.51 & 1.5121 & & 182 & 0.78 & 0.7800 \\
\hline & 213 & 2.93 & 2.9267 & 1.76 & 1.7566 & & 213 & 1.16 & 1.1585 \\
\hline & 243 & 3.61 & 3.6102 & 2.11 & 2.1145 & & 243 & 1.45 & 1.4525 \\
\hline & 274 & 3.83 & 3.8355 & 2.36 & 2.3602 & & 274 & 1.73 & 1.7237 \\
\hline & 304 & 3.81 & 3.8077 & 2.6 & 2.5929 & & 304 & 2.06 & 2.0676 \\
\hline & 334 & 4.62 & 4.6215 & 2.91 & 2.9205 & & 334 & 2.46 & 2.4681 \\
\hline & 355 & 4.84 & 4.8328 & 3.16 & 3.1703 & & 355 & 2.75 & 2.7542 \\
\hline \multirow[t]{9}{*}{318} & 122 & 1.46 & 1.4613 & 0.8 & 0.8012 & \multirow[t]{9}{*}{338} & 122 & 0.44 & 0.4401 \\
\hline & 152 & 2.12 & 2.1224 & 1.25 & 1.2478 & & 152 & 0.5 & 0.5011 \\
\hline & 182 & 2.83 & 2.8299 & 1.66 & 1.6570 & & 182 & 0.75 & 0.7491 \\
\hline & 213 & 3.5 & 3.5034 & 1.97 & 1.9705 & & 213 & 1.43 & 1.4321 \\
\hline & 243 & 4.21 & 4.2041 & 2.73 & 2.7291 & & 243 & 1.77 & 1.7735 \\
\hline & 274 & 4.2 & 4.2001 & 2.88 & 2.8796 & & 274 & 2.43 & 2.4371 \\
\hline & 304 & 4.74 & 4.7470 & 2.93 & 2.9303 & & 304 & 3.08 & 3.0791 \\
\hline & 334 & 5.07 & 5.0600 & 4.09 & 4.0863 & & 334 & 3.51 & 3.5152 \\
\hline & 355 & 6.23 & 6.2183 & 4.36 & 4.3528 & & 355 & 3.95 & 3.9551 \\
\hline \multirow[t]{9}{*}{328} & 122 & 2 & 2.0015 & 0.51 & 0.5104 & \multirow[t]{9}{*}{348} & 122 & - & - \\
\hline & 152 & 2.05 & 2.0484 & 0.85 & 0.8501 & & 152 & 0.41 & 0.4092 \\
\hline & 182 & 2.89 & 2.8923 & 1.41 & 1.4091 & & 182 & 1.06 & 1.0623 \\
\hline & 213 & 4.23 & 4.2346 & 2.22 & 2.2231 & & 213 & 1.25 & 1.2506 \\
\hline & 243 & 4.61 & 4.6104 & 2.93 & 2.9293 & & 243 & 1.95 & 1.9537 \\
\hline & 274 & 5.37 & 5.3625 & 3.26 & 3.2532 & & 274 & 2.9 & 2.8964 \\
\hline & 304 & 7.06 & 7.0543 & 4.33 & 4.3199 & & 304 & 3.5 & 3.4965 \\
\hline & 334 & 8.27 & 8.2566 & 5.12 & 5.1292 & & 334 & 4.35 & 4.3374 \\
\hline & 355 & 9.85 & 9.8661 & 5.6 & 5.6115 & & 355 & 5.16 & 5.1753 \\
\hline \multirow[t]{9}{*}{338} & 122 & 1.65 & 1.6490 & 0.28 & 0.2797 & \multirow[t]{9}{*}{358} & 122 & 0.22 & 0.2201 \\
\hline & 152 & 2.08 & 2.0807 & 0.79 & 0.7904 & & 152 & 0.47 & 0.4698 \\
\hline & 182 & 3.37 & 3.3697 & 1.48 & 1.4817 & & 182 & 1.27 & 1.2696 \\
\hline & 213 & 4.94 & 4.9429 & 2.36 & 2.3610 & & 213 & 2.22 & 2.2249 \\
\hline & 243 & 6.57 & 6.5664 & 3.9 & 3.8957 & & 243 & 2.85 & 2.8429 \\
\hline & 274 & 8.05 & 8.0484 & 4.77 & 4.7655 & & 274 & 3.8 & 3.7971 \\
\hline & 304 & 10.3 & 10.3021 & 6.23 & 6.2419 & & 304 & 4.76 & 4.7709 \\
\hline & 334 & 11.99 & 11.9983 & 7.46 & 7.4527 & & 334 & 5.91 & 5.9237 \\
\hline & 355 & 13.77 & 13.7641 & 8.55 & 8.5453 & & 355 & 6.46 & 6.4405 \\
\hline \multirow[t]{9}{*}{348} & 122 & 1.59 & 1.5910 & 0.24 & 0.2400 & & & & \\
\hline & 152 & 2.03 & 2.0302 & 0.76 & 0.7593 & & & & \\
\hline & 182 & 3.71 & 3.7078 & 1.52 & 1.5190 & & & & \\
\hline & 213 & 6.22 & 6.2236 & 2.88 & 2.8847 & & & & \\
\hline & 243 & 8.01 & 8.0052 & 5.75 & 5.7411 & & & & \\
\hline & 274 & 9.78 & 9.7735 & 7.07 & 7.0802 & & & & \\
\hline & 304 & 12.62 & 12.6362 & 8.28 & 8.2726 & & & & \\
\hline & 334 & 15.22 & 15.2350 & 9.92 & 9.9255 & & & & \\
\hline & 355 & 18.37 & 18.3735 & 11.1 & 11.0985 & & & & \\
\hline
\end{tabular}

\section{Result and discussion}

For the application of the EOSs based models, it is necessary to have knowledge of the molar volume and saturation pressure of dyes, critical temperature, critical pressure and Pitzer's acentric factor of the solute and of solvent.

Saturation pressure of solute can be calculated with Reidel-Plank-Miller Method, critical properties estimated with group contribution method (GCM), Pitzer's acentric factor estimated using Lee-Kesler's Method (Joback et al, 1987, Reid et al, 1987). All estimated critical properties for $\mathrm{D}_{1}, \mathrm{D}_{2}$ and $\mathrm{D}_{3}$ are listed in Table 3 .
Results of calculated solubility corresponding to each experimental data are summarized in Table 2. The optimal fitted binary parameters and the respective AARD combined with the van der Waals mixing and combining rules, with two adjustable parameters (vdW2) and new EOS for $\mathrm{D}_{1}, \mathrm{D}_{2}$ and $\mathrm{D}_{3}$ are presented in Table 4, Table 5 and Table 6, respectively. Good correlation results were obtained between the calculated and experimental solubility, to all fitted models. All fitted models were shown to be able to successfully correlate experimental solubility data. The AARD of new EOS is significantly lower than that obtained from PR-EOS. The mean 
Table 3. Estimated critical and other thermo physical properties of $\mathrm{D}_{2}$

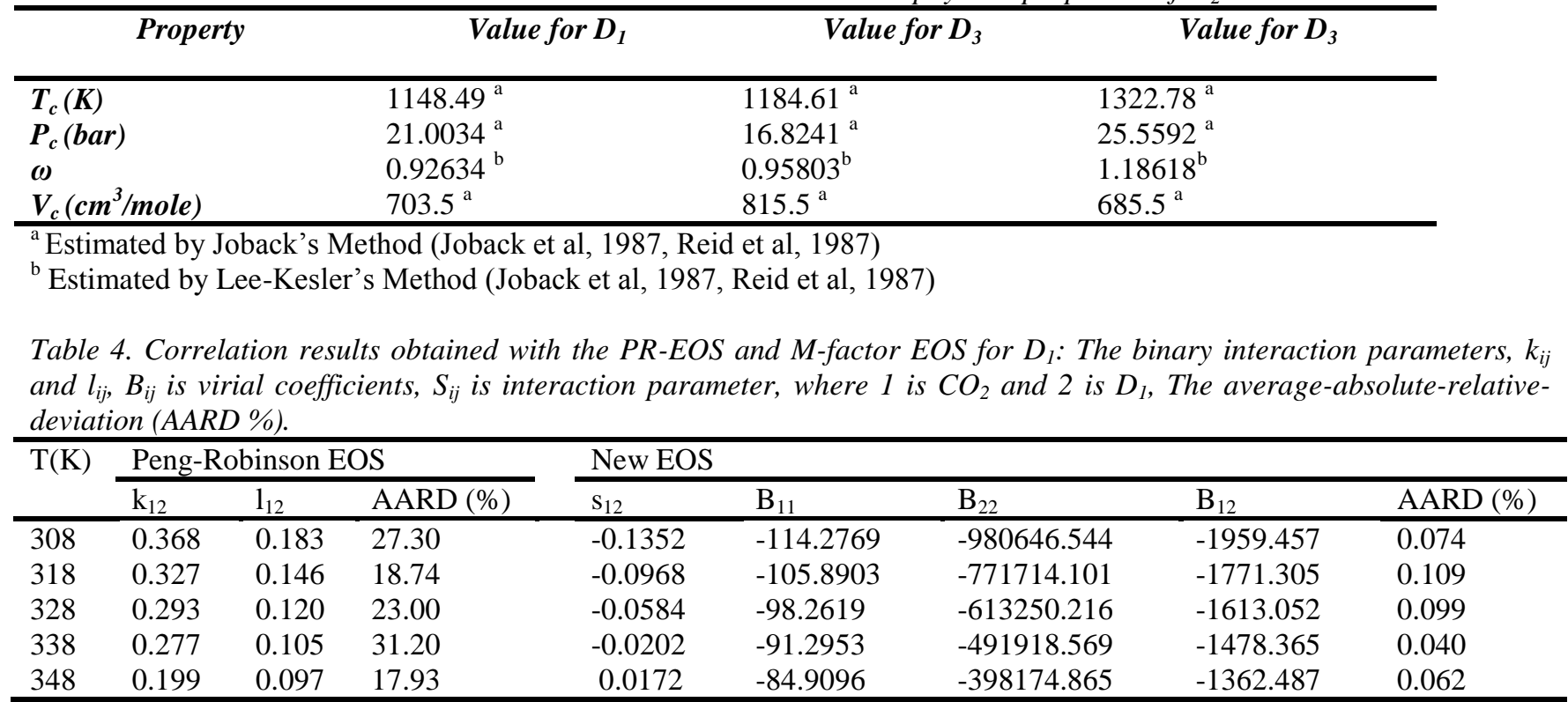

Table 5. Correlation results obtained with the PR-EOS and M-factor EOS for $D_{2}$ : The binary interaction parameters, $k_{i j}$ and $l_{i j}, B_{i j}$ is virial coefficients, $S_{i j}$ is interaction parameter, where 1 is $\mathrm{CO}_{2}$ and 2 is $\mathrm{D}_{2}$, The average-absolute-relativedeviation (AARD \%).

\begin{tabular}{|c|c|c|c|c|c|c|c|c|}
\hline \multirow[t]{2}{*}{$\mathrm{T}(\mathrm{K})$} & \multicolumn{3}{|c|}{ Peng-Robinson EOS } & \multicolumn{5}{|c|}{ New EOS } \\
\hline & $\mathrm{k}_{12}$ & $1_{12}$ & AARD (\%) & $\mathrm{S}_{12}$ & $\mathrm{~B}_{11}$ & $\mathrm{~B}_{22}$ & $\mathrm{~B}_{12}$ & AARD (\%) \\
\hline 308 & 0.427 & 0.201 & 37.00 & -0.1326 & -114.2769 & -1647877.9 & -2456.104 & 0.210 \\
\hline 318 & 0.401 & 0.197 & 25.72 & -0.0958 & -105.8903 & -1293977.5 & -2212.230 & 0.093 \\
\hline 328 & 0.389 & 0.183 & 17.93 & -0.0584 & -98.2619 & -1025817.9 & -2007.930 & 0.127 \\
\hline 338 & 0.377 & 0.176 & 23.33 & -0.0211 & -91.2953 & -820712.79 & -1834.732 & 0.092 \\
\hline 348 & 0.356 & 0.154 & 31.02 & 0.0156 & -84.9096 & -662430.36 & -1686.281 & 0.084 \\
\hline
\end{tabular}

Table 6. Correlation results obtained with the PR-EOS and $M$-factor EOS for $D_{3}$ : The binary interaction parameters, $k_{i j}$ and $l_{i j}, B_{i j}$ is virial coefficients, $S_{i j}$ is interaction parameter, where 1 is $\mathrm{CO}_{2}$ and 2 is $D_{3}$, The average-absolute-relativedeviation $(A A R D \%)$.

\begin{tabular}{|c|c|c|c|c|c|c|c|c|}
\hline \multirow[t]{2}{*}{$\mathrm{T}(\mathrm{K})$} & \multicolumn{3}{|c|}{ Peng-Robinson EOS } & \multicolumn{5}{|c|}{ New EOS } \\
\hline & $\mathrm{k}_{12}$ & $1_{12}$ & $\operatorname{AARD}(\%)$ & $\mathrm{s}_{12}$ & $\mathrm{~B}_{11}$ & $\mathrm{~B}_{22}$ & $\mathrm{~B}_{12}$ & $\begin{array}{l}\text { AARD } \\
(\%)\end{array}$ \\
\hline 328 & 0.399 & 0.187 & 28.01 & -0.0569 & -98.2619 & -2134017.6 & -2092.757 & 0.184 \\
\hline 338 & 0.387 & 0.174 & 31.29 & -0.0234 & -91.2953 & -1695316.1 & -1893.365 & 0.146 \\
\hline 348 & 0.323 & 0.161 & 23.07 & -0.0127 & -84.9096 & -1357802.4 & -1724.621 & 0.181 \\
\hline 358 & 0.301 & 0.143 & 15.86 & 0.0442 & -79.0363 & -1096007.8 & -1580.315 & 0.160 \\
\hline
\end{tabular}

differences between global AARD $(=(\mathrm{AARD}) /$ (total number of data sets)) for the new EOS and PR-EOS are statistically significant. The global AARD values for these data sets are shown in Figure 1. The mean AARD for the new EOS is $0.12 \%$, which is significantly lower than that obtained from PR-EOS $(25.10 \%)$. The new EOS presented more accurate correlation for solubility data in supercritical $\mathrm{CO}_{2}$. It can be employed to speed up the process of SCF applications in industry.

\section{Conclusion}

Solubility data of dyes in supercritical fluids (SCF) are crucial for designing supercritical fluid dying processes. In this study, solubilites of three disperse Azo dyes, 4-(N,Ndimethylamino)-4'-nitroazobenzene $\quad\left(\mathrm{D}_{1}\right), \quad 4-(\mathrm{N}, \mathrm{N}-$ diethylamino)-4'-nitroazobenzene $\left(\mathrm{D}_{2}\right)$ and Parared $\left(\mathrm{D}_{3}\right)$ in supercritical carbon dioxide have been correlated with two equation of state; PR-EOS together with two adjustable parameter van der Waals mixing and combining rules and $\mathrm{M}$-factor EOS. The mean AARD for the M-factor EOS is
$0.12 \%$, which is significantly lower than that obtained from PR-EOS $(25.10 \%)$. The M-factor EOS presented more accurate correlation for solubility data in supercritical $\mathrm{CO}_{2}$. It can be employed to speed up the process of SCF applications in industry.

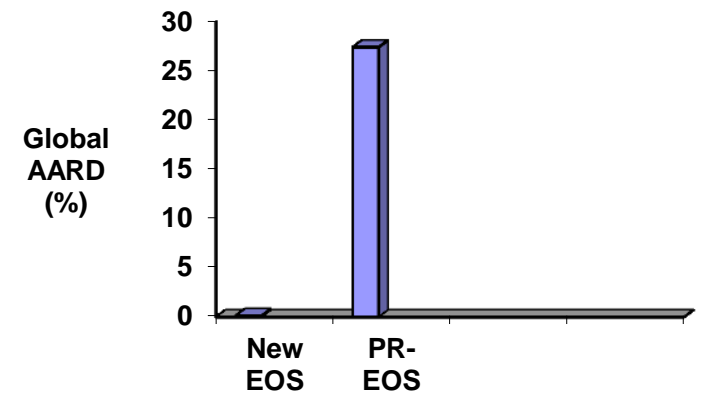

Figure.1. Mean (Global) AARD values for M-factor EOS and PR-EOS. 


\section{Nomenclature List of symbols \\ $\alpha$}

AARD average absolute relative deviation (\%)

B volume parameter in the PR EOS and in the SRK EOS $\left(\mathrm{m}^{3} \mathrm{~mol}^{-1}\right)$

$\mathrm{A}, \mathrm{B}, \mathrm{C}, \mathrm{D}, . . \quad$ virial coefficients

$A^{\prime}, B^{\prime}, C^{\prime}, \quad$ virial coefficients

EOS equation of state

GCM group contribution method

IARD individual absolute relative deviations

$k_{i j} \quad$ binary interaction parameter

$l_{i j} \quad$ binary interaction parameter

M M factor (BP/RT)

$P \quad$ pressure $(\mathrm{Pa})$

PR Peng-Robinson

$R \quad$ ideal gas constant $\left(\mathrm{J} \mathrm{mol}^{-1} \mathrm{~K}^{-1}\right)$

$\mathrm{S} \quad$ Interaction parameter

SCF supercritical fluids

$T \quad$ temperature (K)

$\mathrm{V} \quad$ Volume

$v \quad$ molar volume $\left(\mathrm{m}^{3} \mathrm{~mol}^{-1}\right)$

vdW2 van der Waals mixing and combining rules with two adjustable parameters

y mole fraction

Z compressibility factor

Greek symbols

$\alpha(T), \beta(T), \gamma(T) \quad$ Coefficients of Eq. (22)

$\phi \quad$ Fugacity coefficient

$\rho \quad$ density of pure $\mathrm{CO}_{2}\left(\mathrm{kgm}^{-3}\right)$

$\omega \quad$ Pitzer's acentric factor

$\begin{array}{ll}\text { Subscripts } & \\ 1 & \text { solvent } \\ 2 & \text { solute } \\ \mathrm{c} & \text { critical property } \\ i, j, h, p, k & \text { components } \\ \mathrm{m} & \text { mixture } \\ \mathrm{r} & \text { reduced }\end{array}$

\section{Superscripts}

cal calculated

exp experimental

SCF supercritical phase

sat saturation

\section{References}

Bae, H. K. Hur, B. K. (1996). Solubility of Disperse Dyes in Supercritical Carbon Dioxide. J. KIChe 34, 379-382.

Clifford, A. Bartle, K. D. (1996). Supercritical Fluid Drying. Textile Technol. Int. 6, 113-117.

Cooper, A.I. (2003). Porous Materials and Supercritical Fluids. Adv. Mater. 15, 1049-1059.

Coimbra, P. Gil, M. H. Duarte, C. M. M. Heron, B. M. de Sousa, H. C. (2005). Solubility of a Spiroindolinonaphthoxazine Photochromic Dye in Supercritical Carbon Dioxide: Experimental Determination and Correlation. Fluid Phase Equilib. 238, 120-128.
Draper, S.L. Montero, G.A. Smith, B. Beck, K. (2000). Solubility relations for disperse dyes in supercritical carbon dioxide. Dyes Pigments. 45, 177-183.

Fasihi, J. Yamini, Y. Nourmohammadian, F. Bahramifar, N. (2004). Investigations on the Solubilities of Some Disperse Azo Dyes in Supercritical Carbon Dioxide. Dye. Pig. 63, 161-168.

Haarhaus, U. Swidesky, P. Schneider, G. M. (1995). HighPressure Investigations on the Solubility of Dispersion Dyestuffs in Supercritical Gases by VIS/NIRSpectroscopy Part I. J. Supercrit. Fluids 8, 100-106.

Jafari Nejad, Sh. Abolghasemi, H. Moosavian, M.A. Maragheh, M.G. (2010). Prediction of solute solubility in supercritical carbon dioxide: A novel semi-empirical model. Chem. Eng. Res. Des. 88, 893-898.

Jafari Nejad, Sh. Abolghasemi, H. Golzary, A. Moosavian, M.A. Maragheh, M.G. (2010). Fractional factorial design for the optimization of hydrothermal synthesis of lanthanum oxide under supercritical water condition. $J$. of Supercritical Fluids 52, 292-297.

Jafari Nejad, Sh. Abolghasemi, H. Moosavian, M.A. Maragheh, M.G. (2010). Fractional factorial design for the optimization of supercritical carbon dioxide extraction of $\mathrm{La}^{3+}, \mathrm{Ce}^{3+}$ and $\mathrm{Sm}^{3+}$ ions from a solid matrix using bis(2,4,4- trimethyl pentyl)dithiophosphinic acid + tributylphosphate, Chem. Eng. Res. Des. in press.

Jafari Nejad, Sh. Mohammadikhah, R. Abolghasemi, H. Moosavian, M.A. Maragheh, M.G. (2009). A novel equation of state (EOS) for prediction of solute solubility in supercritical carbon dioxide: Experimental determination and correlation. The Canadian journal of chemical engineering. 87, 930-938.

Janecek, J. Boublik, T. (2003). The Second Virial Coefficient of Polar Rod-Like Molecules. Fluid Phase Equilib. 212, 349-361.

Joback, K.G. Reid, R.C. (1987). Estimation of Pure Component Properties from Group-Contributions. Chemical Engineering Communications 57, 233-243.

Johnston, H. L. Weimer, H. R. (1934). Low Pressure Data of State of Nitric Oxide and of Nitrous Oxide between their Boiling Points and Room Temperature. J. Am. Chem. Soc. 56, 625-630.

Joung, S. N. Shin, H. Y. Yoo, K. P. (1998). Measurement and Correlation of Solubility of Disperse Anthraquinone Azo Dyes in Supercritical Carbon Dioxide. Korean J. Chem. Eng. 15, 78-84.

Jung, J. Perrut, M. (2001). Particle design using supercritical fluids. J. Supercrit. Fluids 20, 179-219.

Kazarian, S.G. (2000). Ploymer processing with supercritical fluid. Polym. Sci. Ser. 42, 78-101.

Kikic, I. Vecchione, F. (2003). Supercritical impregnation of polymers. Curr. Opin. Solid St. M. 7, 399-405.

Meng, L. Duan, Y. Y. Li, L. (2004). Correlations for Second and Third Virial Coefficients of Pure Fluids. Fluid Phase Equilib. 226, 109-120. 
Mohebbi, A. Mohammadikhah, R. (2007). A Simple Equation of State for Calculating the Compressibility Factor of Pure Fluids Based on the Virial EOS, J. Phys. Chem: An Ind. J. 2, 1-6.

Nicola, D. G., Giuliani, G. Polonara, F. Stryjek, R. (2005). Second and Third Virial Coefficients for the R41+N2O System. Fluid Phase Equilib. 228, 373-379.

Pires, A. P. Mohamed, R. S. Mansoori, G. A. (2001). An Equation of State for Property Prediction of AlcoholHydrocarbon and Water-Hydrocarbon Systems. J. Pet. Sci. Eng. 32, 103-114.

Pitzer, K. S. Curl, R. F. (1957). The Volumetric and Thermodynamic Properties of Fluids. III. Empirical Equation for the Second Virial Coefficient. J. Am. Chem. Soc. 79, 2369-2376.

Prausnitz, J. M. Lichtenthaler, R. N. De Azevedo, E. G. (1999). Molecular Thermodynamics of Fluid-Phase
Equilibria, $3^{\text {rd }}$ ed. Prentice Hall PTR, Upper Saddle River, NJ pp.351.

Reid, R.C. Prausnitz, J. M. Poling, B. E. (1978). The Properties of Gases \& Liquids, 4th Edition, Mc GrawHill, New York.

Robinson, D. B. Peng, D. Y. (1976). A New Two-Constant Equation of State. Ind. Eng. Chem. Fund, 15, 59-564.

Saus, W. GmbH, J. (1975). SFD-Dry Dyeing of Polyester in $\mathrm{CO}_{2}$. Textile Technol. Int. 5, 145-150.

Teja, A.S. Eckert, C.A. (2000). Commentary on Supercritical Fluids: Research and Applications. Ind. Eng. Chem. Res. 39, 4442-4444.

Tsonopoulos, G. (1975). Second Virial Coefficients of Polar Haloalkanes. J. AICHE 21, 827-836.

Vetere, A. (1999). An Improved Method to Predict the Second Virial Coefficients of Pure Compounds. Fluid Phase Equilib. 164, 49-59. 